\title{
Mid\#Infrared and Visible Photometry of Galaxies: Anomalously Low Polycyclic Aromatic Hydrocarbon Emission from Low\#Luminosity Galaxies
}

\section{Citation}

Hogg, David W., Christy A. Tremonti, Michael R. Blanton, Douglas P. Finkbeiner, Nikhil Padmanabhan, Alejandro D. Quintero, David J. Schlegel, and Nicholas Wherry. 2005. "Mid\# Infrared and Visible Photometry of Galaxies: Anomalously Low Polycyclic Aromatic Hydrocarbon Emission from Low\#Luminosity Galaxies." The Astrophysical Journal 624 (1) (May): 162-167. doi:10.1086/429686.

\section{Published Version}

doi:10.1086/429686

\section{Permanent link}

http://nrs.harvard.edu/urn-3:HUL.InstRepos:33462886

\section{Terms of Use}

This article was downloaded from Harvard University's DASH repository, and is made available under the terms and conditions applicable to Other Posted Material, as set forth at http:// nrs.harvard.edu/urn-3:HUL.InstRepos:dash.current.terms-of-use\#LAA

\section{Share Your Story}

The Harvard community has made this article openly available.

Please share how this access benefits you. Submit a story.

Accessibility 
The Astrophysical Journal, 624:162-167, 2005 May 1

(C) 2005. The American Astronomical Society. All rights reserved. Printed in U.S.A.

\title{
MID-INFRARED AND VISIBLE PHOTOMETRY OF GALAXIES: ANOMALOUSLY LOW POLYCYCLIC AROMATIC HYDROCARBON EMISSION FROM LOW-LUMINOSITY GALAXIES
}

\author{
David W. Hogg, ${ }^{1}$ Christy A. Tremonti, ${ }^{2}$ Michael R. Blanton, ${ }^{1}$ Douglas P. Finkbeiner, ${ }^{3}$ Nikhil Padmanabhan, ${ }^{4}$ \\ Alejandro D. Quintero, ${ }^{1}$ David J. Schlegel, ${ }^{3}$ and Nicholas Wherry ${ }^{1}$ \\ Received 2004 August 8; accepted 2005 February 14
}

\begin{abstract}
The Spitzer Space Telescope First Look Survey Infrared Array Camera (IRAC) near- and mid-infrared imaging data partially overlap the Sloan Digital Sky Survey (SDSS), with 313 visible-selected $(r<17.6$ mag) SDSS main sample galaxies in the overlap region. The 3.5 and $7.8 \mu \mathrm{m}$ properties of the galaxies are investigated in the context of their visible properties, where the IRAC [3.5] magnitude primarily measures starlight and the [7.8] magnitude primarily measures polycyclic aromatic hydrocarbon (PAH) emission from the interstellar medium. As expected, we find a strong inverse correlation between [3.5]-[7.8] and visible color; galaxies red in visible colors ("red galaxies") tend to show very little dust and molecular emission (low PAH-to-star ratios), and galaxies blue in visible colors ("blue galaxies," i.e., star-forming galaxies) tend to show large PAH-to-star ratios. Red galaxies with high PAH-tostar ratios tend to be edge-on disks reddened by dust lanes. Simple attenuation corrections inferred in the visible bring the visible colors of these galaxies in line with those of face-on disks; i.e., PAH emission is closely related to attenuation-corrected star formation rates inferred in the visible. Blue galaxies with anomalously low PAH-to-star ratios are all low-luminosity star-forming galaxies. There is some weak evidence in this sample that the deficiency in PAH emission for these low-luminosity galaxies may be related to emission-line metallicity.
\end{abstract}

Subject headings: dust, extinction — galaxies: dwarf — galaxies: evolution — galaxies: general — galaxies: ISM — infrared: galaxies

\section{INTRODUCTION}

The Spitzer Space Telescope (Werner et al. 2004) is opening a new window in the universe, with unprecedented sensitivity, angular resolution, and spectral coverage in the mid-infrared and far-infrared. The Spitzer team has made Spitzer's first science data, its First Look Survey (FLS), immediately public. The extragalactic component of the FLS is in a field studied photometrically and spectroscopically in the visible as part of the Sloan Digital Sky Survey (SDSS; York et al. 2000; Abazajian et al. 2004). This overlap permits study of a large sample of visible-selected galaxies with known redshifts and visible spectra in the Spitzer bands.

One of the very exciting capabilities of Spitzer is mid-infrared imaging with the Infrared Array Camera (IRAC; Fazio et al. 2004). For low-redshift galaxies, the longest wavelength channel (centered at wavelength $\lambda=7.8 \mu \mathrm{m}$ ) is most sensitive to largeamplitude, broad emission features in the spectra; these are thought to come from polycyclic aromatic hydrocarbons (PAHs) associated with interstellar dust (e.g., Li \& Draine 2001; Lu et al. 2003; Smith et al. 2004), although this identification is not perhaps iron clad. Since dust is closely associated with star formation, mid-infrared emission is expected to be strongly related to star formation and promises to reveal a lot about galaxy formation and evolution. Indeed, it is and does (e.g., Cohen \& Volk 1989; Roche et al. 1991; Boselli et al. 1998; Dale et al. 2000; Helou et al. 2000; Roussel et al. 2001; Lu et al. 2003; Förster Schreiber et al. 2004; Pahre et al. 2004; Willner et al. 2004).

\footnotetext{
1 Department of Physics, Center for Cosmology and Particle Physics, New York University, 4 Washington Place, New York, NY 10003; david.hogg@nyu .edu.

2 Steward Observatory, 933 North Cherry Avenue, Tucson, AZ 85721.

3 Princeton University Observatory, Peyton Hall, Princeton, NJ 08544.

4 Department of Physics, Jadwin Hall, Princeton University, Princeton, NJ 08544 .
}

The interstellar medium not only traces star formation but also obscures it. PAH emission is expected to be an indirect and nonlinear tracer of star formation activity, but at the same time it is expected to be an order of magnitude less attenuated by dust than the direct measures of young stellar populations in the visible (emission lines) and ultraviolet (thermal continuum). For this reason, Spitzer observations have the potential to measure star formation rates more accurately and to test dust corrections, which depend not just on dust abundance but also on dust grain properties and dust geometry (e.g., Calzetti 2001 and references therein).

Infrared Astronomical Satellite (IRAS) spectroscopy showed that star-forming galaxies have interesting mid-infrared spectra but little diversity; indeed, they showed that PAH emission is very closely related to star formation (Cohen \& Volk 1989; Roche et al. 1991). Star-forming galaxies (spiral and starburst) show strong PAH emission and galaxies without star formation (elliptical and lenticular) do not. The Infrared Space Observatory (ISO) samples of galaxy spectra showed similarly that starforming galaxies show PAH emission (Boselli et al. 1998; Dale et al. 2000; Helou et al. 2000; Laurent et al. 2000; Roussel et al. 2001; Lu et al. 2003; Förster Schreiber et al. 2004) but also showed that low-mass galaxies tended to be deficient in PAH emission relative to what would be expected from an extrapolation from high-mass galaxies with similar specific star formation rates and radiation fields (Boselli et al. 1998). ISO results also suggested that PAH emission is related to metallicity (Sturm et al. 2000).

Along these lines, in the early Spitzer results, one star-forming galaxy with apparently anomalous (termed "extraordinary") midinfrared properties is the $M_{B}=-17.2 \mathrm{mag}$ dwarf galaxy SBS 0335-052, which has metallicity $12+\log (\mathrm{O} / \mathrm{H})=7.338$ (Izotov et al. 1999), about $1 / 20$ the solar value (Allende Prieto et al. 2001). This dwarf galaxy is deficient in PAH emission 
(Thuan et al. 1999; Houck et al. 2004) despite strong star formation activity and substantial dust content. This and other dwarf galaxies demonstrate that galaxies show diverse properties in the Spitzer bandpasses.

For completeness, it is worth noting that relative to normal high-luminosity galaxies, the other population anomalous in midinfrared properties is that of the active galaxies (Roche et al. 1991; Laurent et al. 2000; Sturm et al. 2000), which show much less PAH emission relative to ultraviolet than the star-forming galaxies. Here we look at Spitzer IRAC fluxes from galaxies in the SDSS spectroscopic sample to begin an investigation of the diversity of mid-infrared properties and their relationships with visible properties.

\section{SDSS DATA}

The SDSS data used here are well described in the SDSS Data Release 2 literature (Abazajian et al. 2004 and references therein). The visible photometric measurements on the data used here are discussed in detail elsewhere (Blanton et al. 2003b) and only briefly described here.

The SDSS and Spitzer FLS observations overlap in a roughly $1 \mathrm{deg}^{2}$ field centered on (R.A., Decl. $)=\left(259^{\circ} .5,59^{\circ} .5\right)(\mathrm{J} 2000.0)$. The galaxies used here are from the SDSS main sample (Strauss et al. 2002), which is essentially a flux-limited sample of galaxies with $r<17.6 \mathrm{mag}$. In the overlap of the main sample and the FLS, there are 315 galaxies with redshifts. We removed two galaxies by hand that had very poor SDSS photometry because of "deblending" problems in the software that segments detected objects into unique galaxies. This left 313 galaxies for this study.

The SDSS $g, r$, and $i$ Petrosian (Petrosian 1976) magnitudes are corrected for Galactic extinction with the SFD maps (Schlegel et al. 1998) and $K$-corrected with the KCORRECT V3_2 package (Blanton et al. 2003a), not to redshift $z=0$ but to $z=0.1$, which is roughly the median redshift of this sample. The restframe bandpasses thus made are effectively "blueshifted" by a factor of 1.1 and called ${ }^{0.1} \mathrm{~g},{ }^{0.1} r$, and ${ }^{0.1} i$. Calibration is to the $\mathrm{AB}$ system (Oke \& Gunn 1983). Absolute magnitudes are computed in the ${ }^{0.1} i$ band in the standard way (Hogg 1999), if we assume a cosmological world model with $H_{0}=70 h \mathrm{~km} \mathrm{~s}^{-1} \mathrm{Mpc}^{-1}, \Omega_{M}=$ 0.3 , and $\Omega_{\Lambda}=0.7$.

The SDSS software fits various models to each galaxy image. In what follows we use the axis ratio $b / a$ for the best-fit ellipsoidal exponential model in the observed $i$ band. This fit takes into account the seeing, so the $b / a$ values are in effect seeing corrected.

In each SDSS spectrum, taken through a well-centered circular fiber with a $3^{\prime \prime}$ diameter, $\mathrm{H} \alpha, \mathrm{H} \beta,[\mathrm{O}$ III] $5007 \AA$ (hereafter just $\left[\mathrm{O}\right.$ III]), and [ $\left.\mathrm{N}_{\mathrm{II}}\right] 6584 \AA$ (hereafter just [ $\left.\mathrm{N}_{\mathrm{II}}\right]$ ) line fluxes are measured in $20 \AA$ width intervals centered on each line. Before the flux is computed, the best-fit two-component spectral energy distribution (SED) model spectrum (from Quintero et al. 2004) is scaled to have the same continuum flux as the data in the vicinity of the emission line and subtracted to leave a continuumsubtracted line spectrum. This method fairly accurately models the $\mathrm{H} \alpha$ absorption trough in the continuum, although in detail it underestimates the $\mathrm{H} \alpha$ fluxes by, typically, a few percent (Quintero et al. 2004).

To each galaxy's visible spectrum a model of star formation history and dust attenuation is fitted, providing a visible estimate of the internal attenuation of starlight in the visible due to that galaxy's interstellar medium. We have designed a special purpose code that fits a stellar population model to the galaxy continuum and estimates the attenuation of the starlight under the assumption that any galaxy star formation history can be approximated as a sum of discrete bursts. The library of template spectra is composed of single stellar population models generated by population synthesis (Bruzual \& Charlot 2003). The models incorporate an empirical spectral library (Le Borgne et al. 2003) with a wavelength coverage (3200-9300 $\AA$ ) and a spectral resolution $(\sim 3 \AA)$ that are well matched to that of the SDSS spectral data. The templates include models of 10 different ages $(0.005$, $0.025,0.1,0.2,0.6,0.9,1.4,2.5,5$, and $10 \mathrm{Gyr})$ and three metallicities $\left(1 / 5,1\right.$, and $\left.2.5 Z_{\odot}\right)$. For each galaxy the templates are transformed to the appropriate redshift and velocity dispersion and resampled to match the data. A nonnegative least-squares fit is performed with dust attenuation modeled as an additional free parameter. In the attenuation model the observed flux $F_{\text {obs }}$ is related to the intrinsic flux $F_{i}$ by $F_{\text {obs }}=F_{i} e^{-\tau_{\lambda}}$, where $\tau_{\lambda} \propto \lambda^{-0.7}$ (Charlot \& Fall 2000). The fit of the models to the data is performed from 3600 to $8500 \AA$ in the galaxy frame, with the emission lines masked out. In practice, our ability to simultaneously recover age, metallicity, and attenuation is strongly limited by the signal-to-noise ratio of the data. Hence, we model galaxies as single-metallicity populations and select the metallicity that yields the minimum $\chi^{2}$. (While this is not particularly physical, in practice it is not a bad assumption, since the integrated light of a galaxy tends to be dominated by the light of its most recent stellar generation.)

\section{SPITZER DATA}

The FLS data, like most IRAC data, were taken as a set of hundreds of pointings with the $256 \times 256$ IRAC arrays (with $1.2 \times$ $1.2 \operatorname{arcsec}^{2}$ pixels). Each of these pointings gets passed through the Spitzer basic calibrated data (BCD) pipeline, in which it is flatfielded and calibrated and in which cosmic rays and other bad pixels are identified and flagged.

For each galaxy in the SDSS sample we identified all individual IRAC BCD images in which it appears and performed for each one aperture photometry through 9".2 diameter apertures. Background (or sky level) for each aperture flux was determined by taking a median in an annulus of inner radius $18^{\prime \prime}$ and outer radius $28^{\prime \prime}$. Images with bad pixels or cosmic rays inside the inner aperture (according to the Spitzer-provided mask files) were excised, and the photometry of the remaining images in each band for each galaxy was averaged together (possible because there are multiple dithers per pointing). The measurements were converted to AB magnitudes (Oke \& Gunn 1983).

We applied no $K$-corrections to the Spitzer photometry because the sample spans a small redshift range and, as of this work, there are no reliable $K$-corrections for these spectral ranges available yet. We are working on this presently. Toward the end of $\S 4$ we discuss the effects on our results of the failure to $K$-correct.

\section{RESULTS}

Figure 1 shows the observed Spitzer IRAC [3.5]-[7.8] color as a function of rest-frame visible ${ }^{0.1}[g-r]$ color, with the symbol size (major axis) linearly related to the logarithm of luminosity and the symbol shape showing the axis ratio of the image in the $i$ band. Figure 2 shows the Spitzer color as a function of rest-frame visible absolute magnitude (luminosity). In Figure 1 a clear, strong relationship between visible color and infrared color is seen, with visually red galaxies showing blue infrared colors and visually blue galaxies showing red infrared colors. This trend has a natural explanation: blue galaxies are forming stars, starforming galaxies contain dust, and dust produces PAH emission 


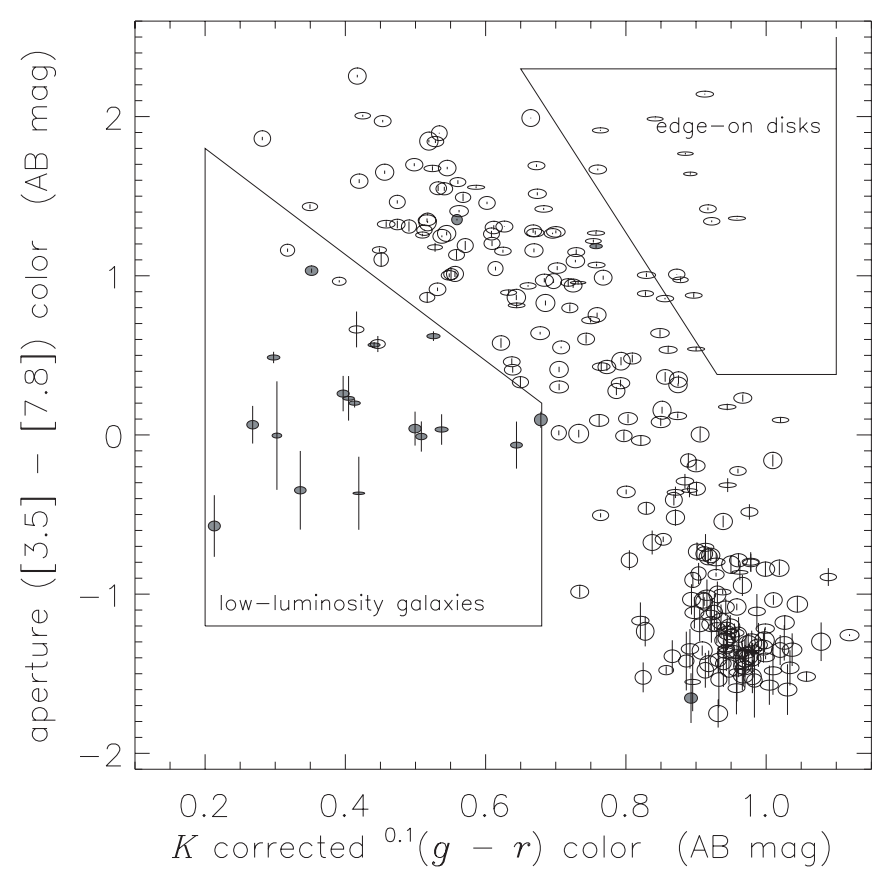

FIG. 1.-Spitzer IRAC observed-frame [3.5]-[7.8] color (aperture fluxes explained in text) as a function of rest-frame visible ${ }^{0.1}[g-r]$ color (blueshifted SDSS bandpasses explained in text). The major axis of the symbol (horizontal extent) is linearly related to the logarithm of the luminosity in the ${ }^{0.1} i$ band (smaller symbols indicate lower luminosities; compare to Fig. 2), and the shape of the symbol shows the galaxy shape as observed on the sky. Symbols for galaxies with very low luminosities $\left(M_{0.1}>-19 \mathrm{mag}\right)$ are filled with gray. Visually blue galaxies tend to have high dust-to-star ratios and therefore red [3.5]-[7.8] colors; visually red galaxies tend to have low dust-to-star ratios and therefore blue [3.5]-[7.8] colors. Exceptions are in the outlined regions: Red galaxies with high dust-to-star ratios tend to be edge-on disk galaxies reddened by their own dust. Blue galaxies with low dust-to-star ratios tend to be low-luminosity galaxies.

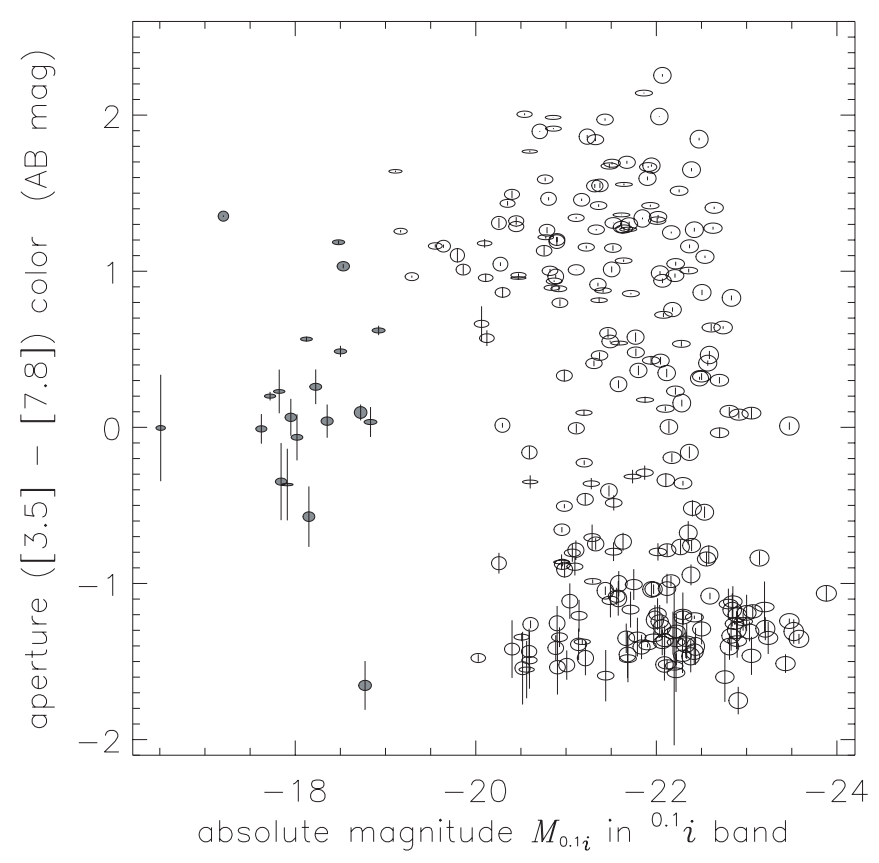

FIG. 2.-Spitzer IRAC observed-frame color as a function of rest-frame visible absolute magnitude (in the $z=0.1$ frame $\operatorname{SDSS}^{0.1} i$ bandpass explained in text). Symbols are the same as in Fig. 1.

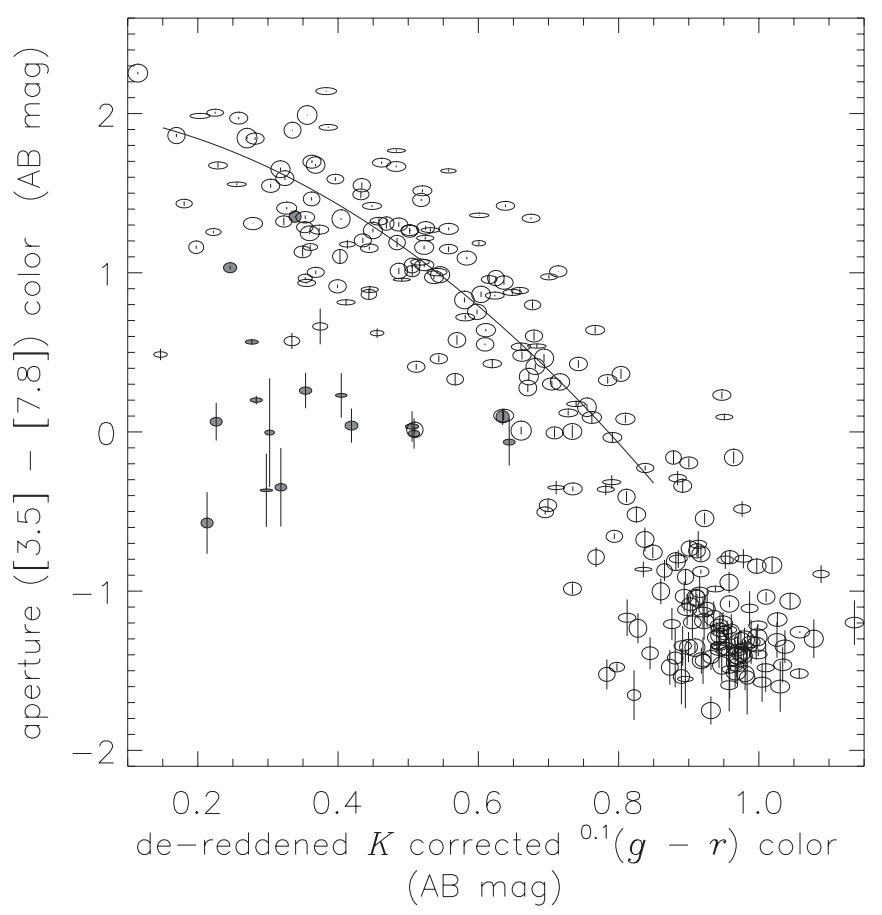

FIG. 3.-Same as Fig. 1, but with reddening corrections applied (see text for details). The edge-on galaxies are no longer outliers from the main trend, but the low-luminosity galaxies remain outliers. Shown also is the best-fit second-order curve, plotted over the ${ }^{0.1}[g-r]$ color range over which it was fitted. The errors were used in the fit, and $4 \sigma$ (and larger) outliers were clipped out.

features in the [7.8] band. Red galaxies are old and dead; they have no material from which to make stars; therefore, they have no dust or PAH features.

Visually red galaxies with red infrared colors tend to be dusty, edge-on galaxies, with high inferred visible attenuations. Figure 3 shows the same data as Figure 1 but dereddened by assuming the best-fit attenuation amplitude (described above) and the trivial $\lambda^{-0.7}$ attenuation law. This procedure is crude and not expected to be correct in detail for any of the sample galaxies.

It is remarkable that the crude and uniform galaxy-by-galaxy attenuation correction does a very good job of placing the visually red but infrared blue galaxies back into the main trend in Figure 1. This is a strong endorsement of the attenuations inferred from the SDSS spectrum analysis and suggests that simple attenuation corrections work well for most galaxies.

Figure 3 also shows a second-order inverse variance-weighted linear fit (first-order fits are not good here) of the infrared [3.5][7.8] color to the visible ${ }^{0.1}[g-r]$ color over the color range $0.15 \mathrm{mag}<{ }^{0.1}[g-r]<0.85 \mathrm{mag}$, with $4 \sigma \sigma$-clipping. At $0.1[g-r]=0.5$, this fit yields the values $[3.5]-[7.8]=1.14 \mathrm{mag}$, slope $-3.19 \mathrm{mag} \mathrm{mag}^{-1}$, and second derivative $-2.80 \mathrm{mag}$ $\mathrm{mag}^{-2}$. The scatter about this fit is about $0.6 \mathrm{mag}$ in the [3.5][7.8] direction, depending on the details of the outlier rejection.

The only remaining outliers on Figures 1 and 3 are visually blue galaxies with bluer than typical infrared colors for their visible color. The symbol sizes on the figures show that these galaxies are low-luminosity galaxies. Evidently, low-luminosity galaxies tend to be deficient in PAH-producing (and visually extinguishing) dust. This fits very well with the results on SBS 0335-052, which show a dwarf galaxy deficient in mid-infrared PAH emission. Indeed, it has been found in visible observations that there is a strong relationship between a disk galaxy's mass and its apparent interstellar medium content or state (Dalcanton et al. 2004). 


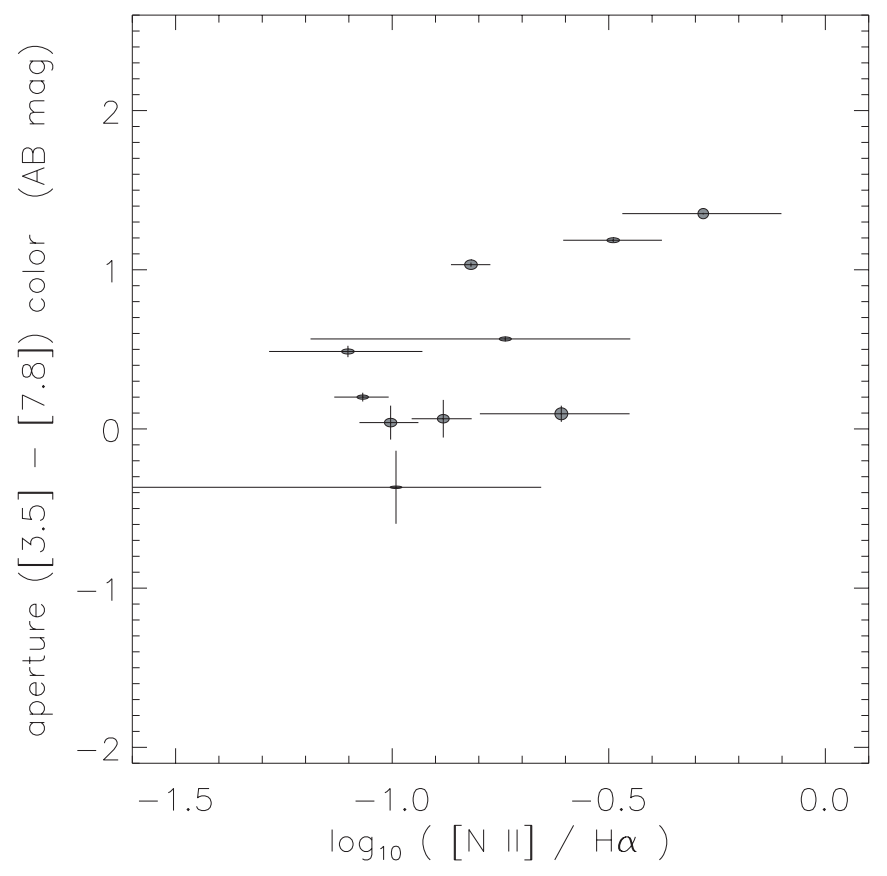

FIG. 4.-Spitzer IRAC observed-frame color as a function of metallicity indicator $[\mathrm{N} \mathrm{II}] / \mathrm{H} \alpha$ (see text for details) for the low-luminosity galaxies, i.e., galaxies with rest-frame visible absolute magnitude $M_{0.1_{i}}>-19.0$, for which the ratio is measured significantly. Symbols are the same as in Fig. 1.

If this effect, namely, anomalous interstellar medium content in low-luminosity galaxies, is due to metallicity, a trend should appear in Figure 4, which shows infrared color as a function of line ratio $[\mathrm{N} \mathrm{II}] / \mathrm{H} \alpha$ for the dwarf galaxies for which that ratio is measured to within a factor of 3. This line ratio is a metallicity indicator (albeit a noisy one, with remaining dependencies on excitation mechanism and ionization parameter) with

$$
12+\log \left(\frac{\mathrm{O}}{\mathrm{H}}\right) \approx 9.12+0.73 \log \frac{[\mathrm{N} \mathrm{II}]}{\mathrm{H} \alpha}
$$

(Denicoló et al. 2002; Kewley \& Dopita 2002). While it is tempting to interpret the figure as showing a dependency of PAH emission on metallicity, given the small sample and large errors, it shows no meaningful trend in this sample. It is worth noting that none of the galaxies in this sample are likely to have metallicities nearly as low as that of SBS 0335-052. Figure 5 is similar to Figure 4 but shows the radiation field hardness indicator $[\mathrm{O} \mathrm{III}] / \mathrm{H} \beta$. No trend is visible.

Figure 6 shows SDSS and IRAC images of some of the lower redshift galaxies in the sample, which are better resolved in the imaging and include low-luminosity members. It is clear that for the high-luminosity galaxies, the [3.5]- [7.8] color is a very good predictor for attenuation-corrected visible color. This is consistent with the hypothesis that for these galaxies [7.8] flux traces star formation and [3.5] flux traces old stellar populations (Pahre et al. 2004). The outliers from this are all low in luminosity; the lowluminosity galaxies tend to be deficient in [7.8] flux.

No $K$-corrections have been applied to the IRAC data. Can the dependence on luminosity in fact be a failure to $K$-correct (since the low-luminosity galaxies are, on average, at lower redshifts)? There is a large, positive PAH feature in most galaxy spectra at around $7.7 \mu \mathrm{m}$ (Li \& Draine 2001; Lu et al. 2003; Smith et al. 2004) that tends to make higher redshift galaxies (and therefore higher luminosity galaxies in this sample) lower in observedframe [7.8] flux. In addition, the low-redshift population in this

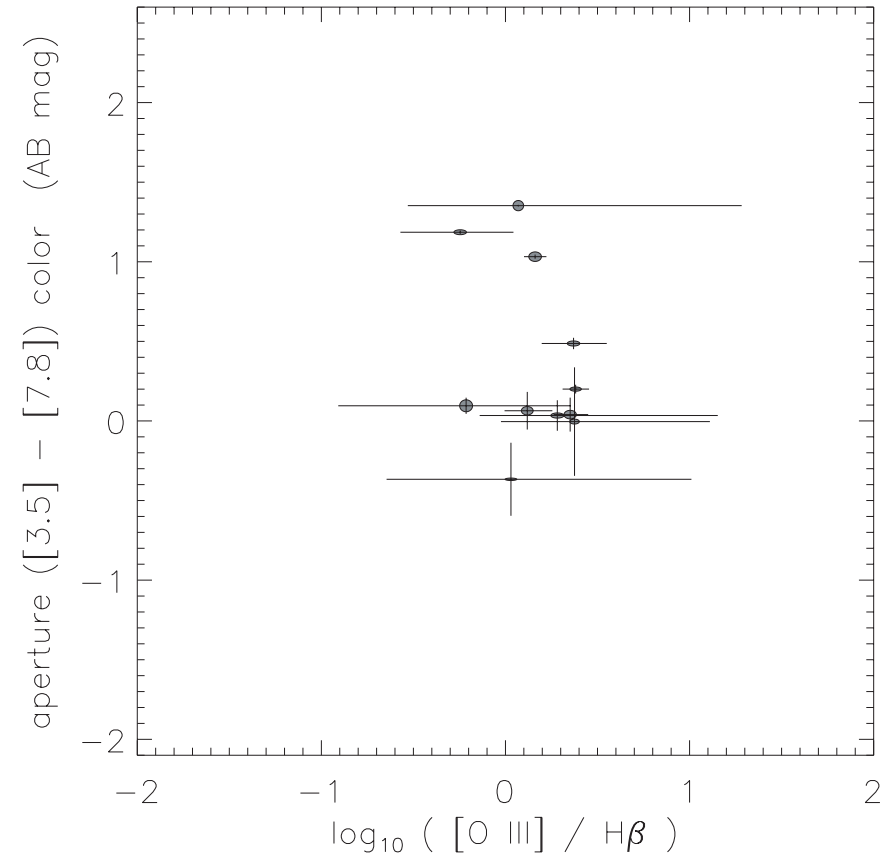

FIG. 5.-Spitzer IRAC observed-frame color as a function of radiation hardness indicator $[\mathrm{O} \mathrm{III}] / \mathrm{H} \beta$ (see text for details) for the low-luminosity galaxies, i.e., galaxies with rest-frame visible absolute magnitude $M_{0.1_{i}}>-19.0$, for which the ratio is measured significantly. Symbols are the same as in Fig. 1.

sample actually spans a large luminosity range (see Fig. 6), within which the luminosity trend of PAH emission is strong. For these reasons we conclude that the trends we see are not due to the failure to $K$-correct.

\section{DISCUSSION}

The principal result of this work is that for a visible-selected sample luminous star-forming galaxies show PAH emission, and old red galaxies do not, in good agreement with previous work from IRAS (Cohen \& Volk 1989; Roche et al. 1991), ISO (Boselli et al. 1998; Dale et al. 2000; Helou et al. 2000; Roussel et al. 2001; Lu et al. 2003; Förster Schreiber et al. 2004), and Spitzer (Pahre et al. 2004; Willner et al. 2004).

This first look at the Spitzer FLS does not show the IRAC photometry "diversifying" the luminous galaxy population beyond what is seen in the visible. In fact, the visible color, shape, luminosity, and spectrum of a luminous galaxy provide an extremely good prediction for its PAH emission and hence its Spitzer IRAC colors. PAH emission is a very good tracer of star formation for these luminous galaxies.

The more surprising result is that low-luminosity galaxies show a deficiency in PAH emission, in agreement with a suggestion from the smaller sample taken by ISO (Boselli et al. 1998). Low-luminosity galaxies also show much more diversity in the PAH-to-star ratio; they clearly provide a very important subject of study for Spitzer. Dwarf galaxies could be PAH-deficient because they tend to be low in metallicity (i.e., they lack the material to make PAHs), because they are low in mass (i.e., they lack the gravitational potential to retain dust and molecules against radiation pressure and winds), because they are young (i.e., they lacked the time necessary to make PAHs), or because they have hard internal radiation fields (i.e., they destroyed their PAHs). Low metallicity is the simplest explanation in many ways and is suggested by the PAH variations within brighter galaxies (Sturm et al. 2000). We have presented weak evidence for a dependence of the PAH-to-star ratio on metallicity, but our metallicity 


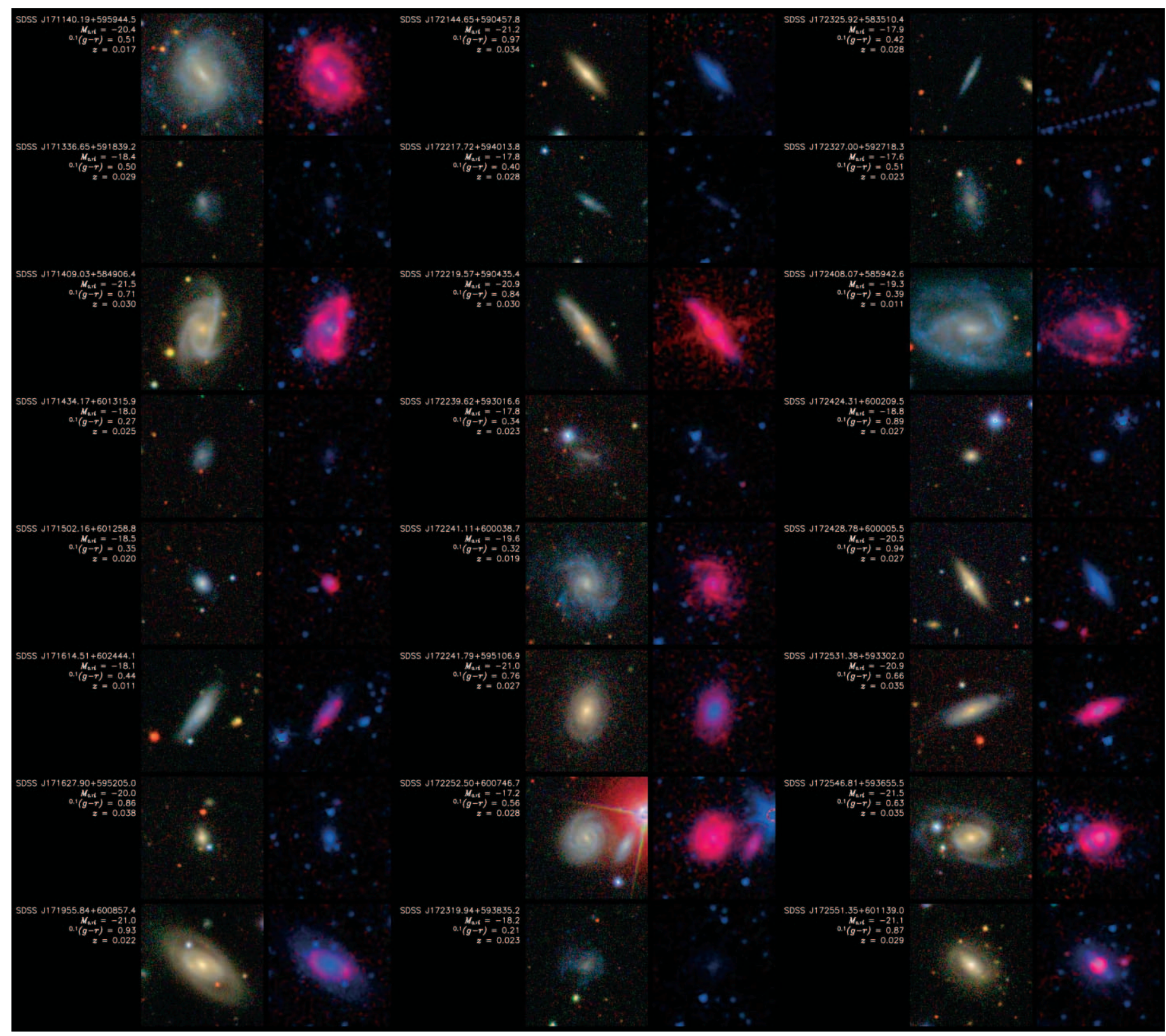

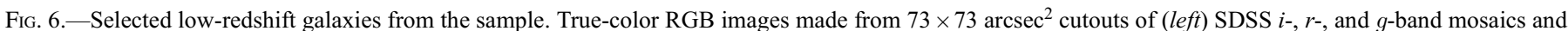
(right) Spitzer IRAC [7.8], [4.5], and [3.5] band mosaics from the Spitzer post-BCD pipeline. The images are made with identical color-preserving stretches (Lupton et al. 2004). In the Spitzer IRAC pictures, stellar light appears blue and interstellar PAH emission appears red.

indicator is poor and is measured for only a small fraction of the sample. The separation of luminosity, excitation, and metallicity effects will be possible with the much larger samples coming from Spitzer Legacy Projects in the near future.

Interestingly, PAH molecules may absorb a significant fraction of all ultraviolet photons in star-forming regions (Dopita et al. 2003). If the PAH abundance is a strong function of metallicity, this will have strong implications for the reionization of the universe at early times, when the star-forming regions had, presumably, lower metallicity than they have today.

Of course, it is also possible that the dwarf galaxies are not PAH-deficient but simply have different interstellar medium geometry (this is suggested by the work on dust lanes in lowluminosity galaxies; Dalcanton et al. 2004) or different relationships between molecules, dust, and radiation fields such that the molecules do not produce apparent emission in the [7.8] bandpass.
The strong, broad spectral features in the mid-IR from PAHs hold great promise for use in photometric redshift determinations (e.g., Simpson \& Eisenhardt 1999). The fact that PAH emission is a strong function of luminosity (or metallicity) may add complications to photometric redshift schemes because not all galaxies are drawn from the same family of SEDs and because there might be significant families of galaxies that lack the highly featured SEDs that are great for redshift inference. Of course any dependence of spectrum morphology on luminosity can also be very useful, since inference of luminosities is often the goal of photometric redshift determinations. Dependencies of SEDs on luminosity are also important for accurate $K$-corrections and bolometric corrections.

Because PAHs are (presumably) formed as stars evolve, the distribution, properties, and demographics of PAHs in galaxies of different types ought to contain information about galaxy formation and evolution; observations of these PAHs will be part of Spitzer's important legacy. 
We thank the entire Spitzer Space Telescope project for starting the mission with a public legacy project. We thank Aaron Barth, Eric Bell, Julianne Dalcanton, Bruce Draine, Rob Kennicutt, Wayne Landsman, Mike Pahre, Bill Reach, and Lisa StorrieLombardi for useful data, discussions, information, or software. We also thank our anonymous referee, who provided many useful references and suggestions.

The Spitzer Space Telescope is a mission of NASA. Funding for the creation and distribution of the SDSS has been provided by the Alfred P. Sloan Foundation, the Participating
Institutions, NASA, the NSF, the US Department of Energy, the Japanese Monbukagakusho, and the Max Planck Society. The Participating Institutions are the University of Chicago, Fermilab, IAS, Japan Participation Group, JHU, LANL, MPIA, MPA, NMSU, the University of Pittsburgh, Princeton University, USNO, and the University of Washington. This research also made use of the NASA Astrophysics Data System. D. W. H., M. R. B., A. D. Q., and N. W. are partially supported by NASA (grant NAG5-11669) and NSF (grant PHY-0101738).
Abazajian, K., et al. 2004, AJ, 128, 502

Allende Prieto, C., Lambert, D. L., \& Asplund, M. 2001, ApJ, 556, L63

Blanton, M. R., et al. 2003a, AJ, 125, 2348 2003b, ApJ, 594, 186

Boselli, A., et al. 1998, A\&A, 335, 53

Bruzual A, G., \& Charlot, S. 2003, MNRAS, 344, 1000

Calzetti, D. 2001, PASP, 113, 1449

Charlot, S., \& Fall, S. M. 2000, ApJ, 539, 718

Cohen, M., \& Volk, K. 1989, AJ, 98, 1563

Dalcanton, J. J., Yoachim, P., \& Bernstein, R. A. 2004, ApJ, 608, 189

Dale, D. A., et al. 2000, AJ, 120, 583

Denicoló, G., Terlevich, R., \& Terlevich, E. 2002, MNRAS, 330, 69

Dopita, M. A., Groves, B. A., Sutherland, R. S., \& Kewley, L. J. 2003, ApJ, 583, 727

Fazio, G. G., et al. 2004, ApJS, 154, 10

Förster Schreiber, N. M., Roussel, H., Sauvage, M., \& Charmandaris, V. 2004, A\&A, 419, 501

Helou, G., Lu, N. Y., Werner, M. W., Malhotra, S., \& Silbermann, N. 2000, ApJ, 532, L21

Hogg, D. W. 1999, preprint (astro-ph/9905116)

Houck, J. R., et al. 2004, ApJS, 154, 211

Izotov, Y. I., Chaffee, F. H., Foltz, C. B., Green, R. F., Guseva, N. G., \& Thuan, T. X. 1999, ApJ, 527, 757

Kewley, L. J., \& Dopita, M. A. 2002, ApJS, 142, 35

\section{REFERENCES}

Laurent, O., Mirabel, I. F., Charmandaris, V., Gallais, P., Madden, S. C., Sauvage, M., Vigroux, L., \& Cesarsky, C. 2000, A\&A, 359, 887

Le Borgne, J.-F., et al. 2003, A\&A, 402, 433

Li, A., \& Draine, B. T. 2001, ApJ, 554, 778

Lu, N., et al. 2003, ApJ, 588, 199

Lupton, R., Blanton, M. R., Fekete, G., Hogg, D. W., O’Mullane, W., Szalay, A., \& Wherry, N. 2004, PASP, 116, 133

Oke, J. B., \& Gunn, J. E. 1983, ApJ, 266, 713

Pahre, M. A., Ashby, M. L. N., Fazio, G. G., \& Willner, S. P. 2004, ApJS, 154, 235

Petrosian, V. 1976, ApJ, 209, L1

Quintero, A. D., et al. 2004, ApJ, 602, 190

Roche, P. F., Aitken, D. K., Smith, C. H., \& Ward, M. J. 1991, MNRAS, 248, 606

Roussel, H., Sauvage, M., Vigroux, L., \& Bosma, A. 2001, A\&A, 372, 427

Schlegel, D. J., Finkbeiner, D. P., \& Davis, M. 1998, ApJ, 500, 525

Simpson, C., \& Eisenhardt, P. 1999, PASP, 111, 691

Smith, J. D. T., et al. 2004, ApJS, 154, 199

Strauss, M. A., et al. 2002, AJ, 124, 1810

Sturm, E., Lutz, D., Feuchtgruber, H., Genzel, R., Kunze, D., Moorwood, A. F. M., \& Thornley, M. D. 2000, A\&A, 358, 481

Thuan, T. X., Sauvage, M., \& Madden, S. 1999, ApJ, 516, 783

Werner, M. W., et al. 2004, ApJS, 154, 1

Willner, S. P., et al. 2004, ApJS, 154, 222

York, D., et al. 2000, AJ, 120, 1579 